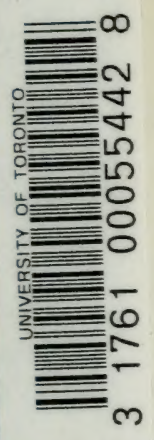

Krug, Wilhelm

Uber die Stellung der

Naturwissenschaft zum LeibSeele-Problem 

Digitized by the Internet Archive in 2011 with funding from University of Toronto 



\section{Über die \\ Stellung der Naturwissenschaft zum Leib-Seele-Problem.}

\section{MAY 141900}

\section{Inaugural-Dissertation zur}

Erlangung der Doktorwürde der

hohen philosophischen Fakultät

der

kgl. bayer. Friedrich-Alexanders-Universität zu Erlangen vorgelegt von

\section{Wilhelm Krug}

aus Hohenwart.

Tag der mündlichen Prüfung: 3. März 1911.

\section{Erlangen.}

K. B. Hof- und Universitätsbuchdruckerei von Junge \& Sohn.

$$
1911 .
$$


Gedruckt mit Genehmigung der hohen philosophischen Fakultät der Universität Erlangen.

Referent: Herr Professor Dr. Hensel. Dekan: Herr Professor Dr. Noether. 
Seinem verehrten früheren Lehrer Herrn Professor Dr. Max Offner in Dankbarkeit gewidmet. 


$$
\begin{aligned}
& B F \\
& 163 \\
& K 78
\end{aligned}
$$

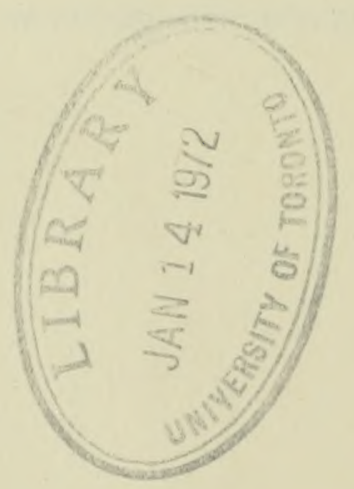




\section{Inhalt.}

Seite

I. u. II. Erkenntnistheoretische Erwägungen über die Art und die Grenzen der naturwissenschaftlichen Erkenntnis 7

III. Die naturwissenschaftliche Erklärung der willkürlichen Bewegung 25

IV. Die mechanistische Naturbetrachtung und das energetische Weltbild Ostwalds . . . . . . 43

V. Qualitative und quantitative, begriffliche und wirkliche Welt . . 55 

I. u. II.

Erkenntnistheoretische Erwägungen iiber die Art und die Grenzen der naturwissenschaftlichen Erkenntnis.

Die immer noch unkritische Auffassungsart von mens (Denken) und corpus (Ausdehnung), die Cartesius an Stelle einer einseitig metaphysischen im Altertum und Mittelalter setzte, schuf in erkenntnistheoretischer Hinsicht keine wesentliche Wandlung zum Besseren. Sie erfuhr mit den empiristischen Arbeiten vor allem der englischen Aufklärungsphilosophie einen entscheidenden Zusammenbruch. Fortan rücken diese Begriffe unter den logisch-erkenntnistheoretischen Klärungsbestrebungen und den psychologischempiristischen Untersuchungen der Philosophie und der philosophierenden Naturwissenschaft in eine ihrer fundamentalen Bedeutung für das Bewußtseinsleben angemessene Beachtung. In dem Wettbewerb um die endgültige Fixierung des mens und corpus und ihrer wechselseitigen Be- 
ziehungen hat die Naturwissenschaft lange Zeit Aussicht mit ihren exakten Methoden, die erstaunlich rasch wertvolle Ergebnisse zeitigen, die Palme zu gewinnen und die Erkenntnisprobleme scheinen glattweg unter Messer und Mikroskop ihrer problematischen Qualitäten verlustig gehen zu sollen. Ein alle möglichen Erfahrungen umklammernder, materialistischer Monismus verknüpft seelische und körperliche Erscheinungen in einem Gesetz und gewinnt sich mit dieser um. fassenden Vereinfachung des Weltbildes immer weitere Anhängerkreise; gleichzeitige, auf metaphysischer und dialektischer Methode aufgebaute Systeme beschränken notgedrungen ihre Gefolgschaft auf Philosophen „vom Fach". Die Weltanschauung um die Mitte des 19. Jahrhunderts droht schließlich mit der Zersetzung aller Spekulation und deren Ersatz durch maßs- und wägbare Gewißheit das Wahrheitsbedürfnis der Nenschheit endgültig befriedigen zu wollen.

Nach einigen Jahrzehnten siegesgewissen Vowärtsstürmens auf allen Gebieten geistiger Betätigung beginnt jedoch eine unerwartet ein. setzende Ernüchterung Platz zu greifen. Die mannigfachen Bedenken, welche durch die weit voneinander abweichenden und sich gegenseitig ausschlickenden philosophischen Anschauungen der großen Denker bei den Naturforschern grol gezogen worden sind und sie daher zu ihrer fundamentalen Methodenänderung veranlabt haben, 
schleichen sich in ihre eigene Reihen. Das Rätsel der Erkenntnis, das Kant mit seinem Transzendentalismus und Fichte mit seiner Wissenschaftslehre zu lösen unternommen, das Schelling mit der Vernichtung alles Dualismus und dem Axiom der "totalen Indifferenz von Subjekt-Objekt" und Hegel durch eine logisch-metaphysische Entwicklung von "Sein, Wesen u. s. w. " im „System der Philosophie" des Rätselhaften zu entkleiden suchte; das später Herbarts Wissenschaft vom Realen sowie Schopenhauers Methode der unmittelbaren Anschauung mit neuen Gesichtspunkten in Angriff genonmen, dieses Rätsel ist nunmehr auch der Naturwissenschaft als immer noch der Aufklärung bedürftig zum Bewußtsein gekommen und allmählich vollzieht sich in ihren Einzeldisziplinen das Geständnis eigenen Versagens und damit die Einsicht von der Berechtigung der ehemals zur Seite gesetzten philosophischen Lösungsversuche. Selbst zu philosophieren bequemt sie sich hinfort und neben $\mathrm{Maß}$ und Gewicht besteht für sie Erkenntnistheorie.

Die Zurücknahme der vordem so leichthin sanktionierten materialistischen Relation zwischen corpus und mens, zwischen körperlichem und geistigem Vorgang, sowie die äußerste Vorsicht in der Wahl neuer Gesichtspunkte und Theorieen ist die erste Folge jener Reaktion. Die einseitige Abhängigkeit der seelischen Vorgänge ron den körperlichen, beziehungsweise die Gleich- 
setzung beider Arten als mechanischer verliert den Nimbus der Selbstverständlichkeit, der ihnen lange Zeit angehaftet hat; die bisher als philosophisch verpönten Anschauungen des Parallelismus und der gegenseitigen Kausalitätsbeziehung werden dagegen in den Bereich mindestens der Möglichkeit gezogen. Vor allem aber ringt sich die Erkenntnis durch, daß auch der Naturwissenschaft trotz eines fast unübersehbaren Gebiets. umfanges zu respektierende Grenzen gesetzt sind, über die hinauszugehen ihr die blof im Begrifflichen gründende Art ihrer Erkenntnisse und die Beschaffenheit ihrer Hilfsmittel nicht gestattet. Die Einsicht in die Gebote und Verbote ihrer Aufgabe, in das Wesen ihrer Erkenntnis, zeitigt in dieser Hinsicht für die Naturwissenschaft üherraschende Ergebnisse.

Welches ist die Aufgabe der Naturwissenschaft? Beschreibung? Erklärung? Mit dem Erfassen der Tragweite des Wortes Erklärung, womit wir Zweck und Grund und innerstes Wesen etwa eines physiologischen oder psychologischen Vorgangs dargestellt wissen wollen, ist sofort die Einsicht gegeben, daß unter den erreichbaren Zielen der Naturw issenschaft eine restlos befriedigende Erklärung nicht in Frage kommen kann. Die Erklärung der Natur. wissenschaft wird niemals cine radikale sein und sich immer mehr oder minder einer logischen Annäherung an die Be. 
schreibung bequemen müssen; eine Notwendigkeit, die sich, wie wir desweiteren noch sehen werden, aus dem Wesen des naturwissenschaftlichen Erkennens ergibt.

Was die Naturwissenschaft erzielt, bezw. zu erstreben sucht, ist Sammlung und Ordnung von Begriffen und darauf aufgebauten Lehrsätzen zu einem nach formalen Regeln konstruierten Gebäude. Mit dessen Vollständigkeit und Einheit, die sie durch planmäßige Verknüpfung generisch verwandter Erkenntnisse erhält, ist ihre Tätigkeit abgeschlossen. $\mathrm{Ob}$ diese Systematik nun hauptsächlich auf logischer Unter- und Überordnung, auf Klassifikation beruht, oder das Grundund Folgeverhältnis als leitendes Motiv zugrunde liegen hat, das logische Prinzip ist immer ihr Charakteristikum.

Indiziert nun der logische Zusammenhang, der mit innerer Notwendigkeit von Begriff zu Begriff, von Gesetz zu Gesetz uns forttreibt, irgendwie eine objektive Wirklichkeit? Vielleicht die Existenz realer Gegenstände, die mit jenem Zusammenhang eindeutig bestimmt sind, oder realer Gesetzmäßigkeit, wonach mit immanentem Zwang sich Vorgänge folgen? Oder ist damit bloß eine einfache Registrierung der Konstanz gewisser Geschehnisse und der Stetigkeit der Qualitäten gewisser Erscheinungsformen gegeben? Mit anderen Worten: sind mit der apodiktischen 
Gewißheit und Gültigkeit der logischen Vernunftgesetze auch inhaltlich unumstößliche Naturgesetze gewonnen?

Betrachten wir das Gesetz der Erhaltung des Stoffes (der Konstanz der Masse) in einem konkreten Beispiel!

Mit der Verbrennung einer Kerze im abgeschlossenen Raum erfolgt infolge Sauerstoffentzuges aus der abgeschlossenen Luft durch die Flamme einerseits, infolge der Veründerungen an der Kerze (Verkohlung des Fadens u.s.w.) andererseits, eine ohne weiteres erkennbare Massenände. rung. Die Gewichtsprobe der im abgeschlossenen Raum befindlichen Substanzen ergibt vor und nach dem Verbrennungsprozeß den gleichen Be. fund; die getrennte Abwage von Kerze, bezw. Luft vor, den Residuen der Kerze, bezw. übriggebliebener Luft $\mathrm{n}$ ach dem Verhrennungsprozeß jedoch ergibt ein Plus an Masse der Kerzenresiduen um das Gewicht des der Luft entzogenen Sauerstoffes. Ein entsprechendes Resultat ergiht sich bei allen Verbrennungs-, wie anderen chemischen und physikalischen Prozessen.

Ergebnis: Die Masse der bei beliebigen physischen Vorgängen beteiligten Körper bleibt unverändert, durch Induktion verallgemeinert: die Gesamtheit aller existierenden Maße ändert sich nicht.

Ist damit nun ein - formell und materiell - völlig zureichendes Gesetz 
gewonnen, das mit apodiktischer Gewiß heit jegliche Abweichung für alle $\mathrm{Zu}$ kunft ausschließen würde? Wie nun, wenn die Molekulartheorie, das Fundament unserer Chemie und Naturwissenschaft, sich für die $\mathrm{Zu}$. kunft nicht mehr halten ließe; wenn das Massengesetz, dem jene Theorie die Stütze verleiht, unter ein allgemeineres Energiegesetz zu stehen käme, nach welchem bei beliebigem Austausch aktueller und potentieller Energie die Summe beider eine konstante ist? "Es würde dann vorkommen können, daß Energie von der Art, die wir Materie nennen, sich in Energie, die nicht Materie ist, verwandelt, und die Konstanz der Masse wäre dann durchbrochen. Gegenwärtig hat man schon an drei auseinanderliegenden Stellen Spuren solcher Möglichkeiten entdeckt, erstens Anzeichen von (freilich minimalen) Gewichtsänderungen bei chemischen Umsetzungen, zweitens Anzeichen dafür, daß uns Masse vorgetäuscht werden kann, wo es sich vielleicht nur um abstrakte Bewegungen handelt (die "scheinbare Masse" strahlender Teilchen) und drittens Anzeichen merkwürdiger Beziehungen zwischen Materie und Energie bei dem Phänomen der Radioaktivität ..."1).

Das Hypothetische der Naturwissenschaft ist damit gegeben. Ihre Erkenntnisse, die auf den

1) F. A uerbach, Die Grdbegr. d. mod. Naturl. S. 94. 
durch Denken - durch Schlüsse - verarbeiteten Beobachtungen beruhen und im durchgehenden Reduktionsprozeß, d. i. der Zurückführung neuartiger und komplizierter Vorgänge auf immer einfachere, umfassendere Gesetze, der Aufdeckung des allgemeinen Prinzips zustreben, entbehren nach ihrer inhaltlichen Seite der unmittelbaren Evidenz der Grundsätze und Folgesïtze der Logik (und Mathematik), sie sind jederzeit nach ihrer materiellen Seite hin der Gefahr einer notwendig werdenden Modifizierung preisgegeben. Was Unwandelbares an ihnen ist, die Begriffe, die Ordnungen, die Beziehungen, ist Form, ist Anteil des Denkens, das völlig unabhängig von bestimmter Materie besteht.

Bei absoluter formaler Wahrheit eines Erkenntnisses besitzt darum die Naturwissenschaft noch nicht die Gewißheit der materiellen.

Ein weiteres ist hier noch zu berüchsichtigen. Die inhaltliche Richtigkeit irgendeiner bestimmten Erkenntnis vorausgesetzt, sieht sich die Naturwissenschaft vor die neue Frage gestellt, ob jene Wahrheit nun phänomenolngische oder reale Unterlagen besitze.

Der Entscheid in diesen letzten Problemen, der lange Zeit den metaphysischen Spekulationen über das Wesen des Denkens und Seins überlassen war, ist von fundamentaler Bedeutung für die Naturwissenschaft, nachdem sie sich in psychologische und erkenntnistheoretische Domïnen be. 
geben und daher selbst größtes Interesse an den die Wesensunterschiede und die Beziehungen zwischen körperlichen und geistigen Vorgängen betreffenden metaphysischen Untersuchungen nehmen muß. Während die Naturwissenschaft nun früher diese Fragen aus eigener Kraft, mit Beiseitesetzung erkenntnistheoretischer Erwägungen zu lösen gesucht, ist sie sich heute in der großen Mehrzahl ihrer Vertreter - in Erkenntnis des Wesens und der Aufgabe der Naturwissenschaft - der Beschränkung der ihr zu Gebote stehenden Hilfsmittel bewußt geworden und macht sich jene der Gegnerseite von ehemals zunutze. Sie stützt sich aber auch mit Erfolg auf die Vorzüge der ihr speziell eigenen Methoden in den Streitfragen, die sie mit der Philosophie im allgemeinen und mit der Metaphysik im besonderen gemein hat. Die folgenden Ausführungen über die Stellungnahme der neueren Naturwissenschaft zum Problem der Beziehungen zwischen Körper und Geist werden uns hiervon eine Anschauung geben.

II.

Der schon oben gezeichnete Standpunkt der Naturwissenschaft um die Mitte des 19. Jahrhunderts war ein extrem materialistischer, der unter der Wirklichkeit nur Körper und körper. liche Tätigkeit befaßte. Mit dem materiellen 
Aufschwung um 1850 hatte sich ein gleichzeitiger der Naturwissenschaften vollzogen und nun „ergreifen Chemiker und Physiologen die Kelle der Metaphysik". Bezeichnend für die lebhafte philosophische Strömung in der Naturwissenschaft vor allem der Zeit des Materialismusstreites ist auch der küstliche Satz, „daß in Deutschland der Apotheker kein Rezept anfertigen kann, ohne sich des Zusammenhangs seiner Tätigkeit mit dem Universum bewuft zu sein" "). Moleschott, Vogt, Büchner sind die Vorkämpfer der materialistischen Richtung. Die hergebrachte Philosophie bekommt üble Fußsschritte. „Philosphische Ausführungen, welche nicht von jedem Gebildeten begriffen werden können, verdienen nach unserer Ansicht nicht die Druckerschwärze, welche man daran gewendet hat." So Büchner, der sich aber trotz dieses anmaßenden Urteils die Definition erlaubt "Hypothese bedeute jede Art von ungerechtfertigten Annahmen, wie z. B. die deduzierten Sätze der philosophischen Spekulation". Und Moleschotts erkenntnistheoretische Qualititen verhindern ihn nicht, aus dem Umstand, daß all unser Wissen ein gegenständliches ist, nun auch schon mit den Dingen für uns die Dinge an sich als gegeben zu setzen ${ }^{2}$ ). Czolbe, angeregt durch die Lotzesche Besei-

1) Lange, Gesch. d. M. S. 120.

2) Ebenda, S. 136 . 
tigung des „übersinnlichen Begriffs" der Lebenskraft, setzt sein Bestreben in die Beseitigung aller transzendenten Kräfte und Wesen, in den Bakonischen Ausschluß alles Übersinnlichen; dabei gelingt es aber ihm so wenig wie Feuerbach, Vogt und Moleschott, den Bereich der gemeinsam bekämpften spekulativen Philosophie zu verlassen, und $\mathrm{C}$ zolbe sieht sich schlieflich zum Bekenntnis genütigt: „scheint es mir doch selbst, daß ich durch die Konsequenzen, zu denen das Prinzip mich zwingt, in eine märchenhafte Gedankenwelt geraten bin ${ }^{41}$ ).

Die radikale Form des Materialismus, die durch Czolbe schon eine beträchtliche Milderung erfährt, hat unter den Naturforschern allmählich ihre Anhänger verloren. Der La p lac esche Geist mag immerhin nach den Regeln der atomistischen Mechanik Vergangenheit und Zukunft des Universums in eine Rechnung stellen und mit „astronomischer" Kenntnis der Gehirnvorgänge einen Einblick in die nervösen Unterlagen der Bewußtseinsvorgänge gewinnen, er ist dennoch weder imstande, die Atome selbst $\mathrm{zu}$ verstehen, noch mit der Bewegung der Atome die einfachste biologische oder psychische Tatsache zu erklären. Früher glaubte die Naturwissenschaft nur der zeitlichen Entwicklung ihrer Forschung zu bedürfen, um schließlich alle körperlichen - und

1) Ebenda S. 149. 
Bewußtseinserscheinungen auf physikalische, bezw. chemische Gesetze zurückzuführen. Nun scheint es Bunge dagegen ${ }^{1}$ ), „daf die Geschichte der Physiologie genau das Gegenteil lehre: Je eingehender, vielseitiger, gründlicher wir die Lebenserscheinungen zu erforschen streben, desto mehr kommen wir zur Einsicht, daß Vorgünge, die wir bereits geglaubt hatten, physikalisch und chemisch erklären zu können, weit verwickelterer Natur sind und vorläufig jeder mechanischen Erklärung spotten."

Also schon eine Grenze für den Laplaceschen Geist!

Im gesamten Gebiet der Biologie besteht danach keine Erscheinung, die restlos auf physikalische oder chemische Tatsachen zurückzuführen wäre.

Es gelingt weder die Erscheinungen der Resorption und Sekretion endosmotischen Gesetzen zu unterstellen, noch die auf dem Prinzip der Arbeitsteilung erfolgende Differenzierung der Gewebezellen rein mit chemischen Umwandlungen zu begründen. Die hydrodynamischen Gesetze reichen nicht aus, die Möglichkeit der Blutzirkulation verständlicher zu machen, noch aerodynamische u. s. w. die Möglichkeit der Respiration $z u$ demonstrieren. Es reicht aber auch - zu diesem Geständnis mufis der Naturforscher sich

1) G. Bunge, Lehrb. d. phys. u. path. Chemic, S. 5. 
bequemen - keine mechanische Erklärung hin, nur die einfachsten Funktionen der Bewegung oder Ernährung einer einzigen Zelle zu deuten. „In der kleinsten Zelle - da liegen schon alle Rätsel des Lebens vor uns und bei der Erforschung der kleinsten Zelle - da sind wir mit den bisherigen Hilfsmitteln an der Grenze angelangt" 1 ).

Immerhin beruht die Vorsicht, die die Naturwissenschaft gegenüber den biologischen Erscheinungen anzuwenden hat, nicht auf derart ausgeprägten Gegensätzen, wie sie zwischen den Vorgängen physischer und psychischer Art konstatiert werden müssen. Wir sind heute berechtigt, wie wir weiter unten noch ausführen werden, die biologischen Vorgünge unter gewissen Voraussetzungen in eine Parallele mit den übrigen physischen Vorgängen zu stellen ${ }^{2}$ ).

1) Bunge, Lehrb. .... S. 12.

2) Interessant sind zwei von R. Hertwig bei Gelegenheit seiner Rekt.-Antrittsrede (W.-S. 1910/11) erwähnte neuere biologische Forschungsergebnisse: „1. Im Anschluß an die Untersuchungen Quinckes hat $\mathrm{O}$. Bütschli gezeigt, daß der mikroskopische Bau des „sogenannten homogenen Protoplasmas" eine große Ähnlichkeit besitzt mit dem mikroskopischen Bau von Śchäumen, wie man sie erzielt, wenn man ein außerordentlich fein verteiltes Gemenge zweier nicht miteinander sich mischender Flüssigkeiten - z. B. Olivenöl und Pottasche Lösung - erzielt. Derartige Gemenge zeigen nicht nur ähnliche Bewegungserscheinungen wie das Proto- 
Die Kluft $\mathrm{z}$ wischen Physischem und Psychischem ist dagegen für die Naturwissenschaft immer noch unüberbrückbar. Du Bois-Reymond hatte es sich vor allem zur Aufgabe gemacht, gegenüber der materialistischen

plasma, sondern auch eine Reibe weiterer Übereinstimmungen. Die feineren Strukturen, welche das Protoplasma besonders bei den Zellteilungen annimmt, galten lange Zeit mit der Annahme einer flüssigen Beschaffenheit unrereinbar. Nun hat aber Rhumbler nachgewiesen, daß das Protoplasma die physikalischen Eigenschaften ron Flüssigkeiten besitzt. Daher ist es wichtig, daß man experimentell an Flüssigkeitsgemengen analoge Strukturen herrorrufen kann, wie sie als Beweise gegen den flüssigen Aggregatzustand des Protoplasma galten. 2. In der Neuzeit hat man den Ablauf der Lebensprozesse der Zelle bei wechselnden Temperaturen untersucht und gefunden, daß innerhalb gewisser Grenzen die Temperatursteigerung eine Beschleunigung erfährt, welche sehr :ihnlich ist der Beschleunigung, welche chemische Realtionen durch Temperatursteigerungen gemäß dem Van't Hoffschen Gesetz erfahren. - Es ist klar, daß, je zahlreicher und intimer die Analogien zwischen chemisch-physikalisehen und biologischen Vorgängen werden, um so mehr die Auffassung, daß diese auf jene ohne Rest zurückführbar sind, an Wahrscheinlichkeit gewinnt (vgl. Peter, Arch. Entw.Mech. Bd. 20, Marcus, ebenda Bd. 22, Hertwig, Arch. mikr. Anat. Bd.51). Daß aus der weitgehenden Teilbarkeit der lebenden Substanz, spezieller einzelliger Leberesen und aus den zweekmäßigen Regulationen äußeren Eingriffen gegenüber einer mechanischen Auffassung des Lebens große Schwierigkeiten erwachsen, läßt sich nicht leugnen; ich halte es aber nicht für erwiesen, daß diese Schwierigkeiten, wie D riesch meint, unüberwindbar sind" (S. 32, Anm. 6). 
Naturforschung zu betonen: „daß nicht allein bei dem heutigen Stand unserer Kenntnis das Bewufitsein aus seinen materiellen Bedingungen nicht erklärbar ist, was wohl jeder zugibt, sondern daß es auch der Natur der Dinge nach aus diesen Bedingungen nie erklärbar sein wird" (Gr. d. Naturerk. S. 20).

Keine Gehirnforschung enthüllt uns geistige Vorgänge und keine Kenntnis von den materiellen Grundlagen der Vorstellungsassoziationen unterrichtet uns (wenn auch über Bedingungen) über das Zustandekommen des Geisteslebens ${ }^{1}$ ).

So waren "Grenzen des Naturerkennens" auch der Naturwissenschaft zum Bewußtsein ge-

1) Immerhin finden sich noch immer nicht wenige, niemals zu überzeugende Naturwissenschaftler. So meint A. Forel: Die Seelentätigkeit miisse aus der Tätigkeit premitiver Zellen hervorgegangen sein, da sie doch nur zusammengesetzte Neuronentätigkeit sei („Gehirn und Seele“, 1910, S. 77). - Weiter: „Daß . . ein Rest für eine energielose Seele oder für eine seelenlose Gehirnenergie übrig bleibe, wird sicher kein vorurteilslos (\%) denkender Leser finden können" (S. 87). - Ebenda: Die Gegner der „so gewonnenen Identitätstheorie zwischen unseren oberbewußten Seelenerscheinungen und der Energetik des Großhirns" können sich immerhin ,den Spaß machen, das Weltall mit leeren Worten, wie Substanz, Idee, Wille, Monaden, Alleins, Unbervußtes, Absolutes, Ding an sich, Subjekt-Objekt, Götzen u. dergl. m. zu konstruieren". Hier vergißt wohl Forel auf jeden Fall, in die Reihe der ron ihm angeführten ,leeren Worte" auch seinen monistischen Energiebegriff einzuweisen. 
kommen, nachdem sie lange Jahre über die Philosophie hinweg ihre eigenen Bahnen eingeschlagen und in Auferachtlassung erkenntnistheoretischer Elementarfragen mit eigenen Hilfsmitteln die letzten Probleme zu lösen gesucht hatte. Was lange Zeit als verpönt galt, als Naturforscher philosophisches Terrain anzuerkennen, wird jetzt zum Zwang und das erkenntnistheoretische Geständnis von Grenzen der Naturerkenntnis, die durch die denklsar größten wissenschaftlichen Fortschritte nicht überschritten werden können, drängt sich auch Chemikern und Physiologen auf.

„.. . naturwissenschaftlich erkennen lsönnen wir ein für allemal nur die Symptome und Bedingungen des Geistigen außer uns, nicht dieses selbst" 1), ist eine der Tatsachen, die der Naturwissenschaft zu denken geben müssen, deren Anführung Lange bald eine zweite, nicht minder wichtige folgen läßt: Läßt sich eine transzendente Spekulation von der Hand weisen, „so bleibt doch der Boden, auf dem diese Spekulation erwachsen, und in negativer Hinsicht können wir mit Zuversicht antworten: von der toten, stummen und schweigenden Welt der schwingenden Atome wissen wir nichts, als $\mathrm{da} \beta$ sie eine notwendige Vorstellung für uns ist, insofern wir den Kausalzusammenhang der Erschei-

1) F. A. Lange, G. d. M. II, S. 205. 
nungen in wissenschaftlicher Weise darstellen wollen. Da wir aber auf einem Punkte gesehen haben, daß diese notwendige Vorstellung nicht das Gegebene, nämlich unsere Empfindungen, sondern nur eine gewisse Ordnung im Entstehen und Vergehen derselben erklärt, so müssen wir einsehen, daß diese Vorstellung nach ihrer ganzen Natur und ihren notwendigen Prinzipien nicht geeignet ist, uns das letzte, innerste Wesen der Dinge zu enthüllen $\left.{ }^{1}\right)$."

Für den Naturforscher ergeben sich aus den soeben geschilderten Erkenntnissen, zu denen er sich genötigt sieht, zwei fundamentale Geständnisse :

Erstens es existiert neben den körperlichen Vorgängen, neben Druck und Stok, bezw. Energie noch eine geistige Welt, die generell von der körperlichen sich unterscheidet;

zweitens ist es der Naturwissenschaft über diese, wie über jene, Wesens erkenntnisse zu schaffen unmöglich.

Über letztere Behinderung wird sie sich ohne weiteres hinwegsetzen. Eine Welt, die mit ihren Methoden nicht bearbeitet werden und daher als Forschungsziel nicht in Betracht kommen kann, überläßt sie von vornherein der Transzen-

3) Lange, G. d. M., S. 212. 
denzphilosophie als Objekt der Erkenntnis. Denn ein innerer Kern einer Erscheinung, ein metaphysisches Ding an sich, ist nicht sichtbar, nicht tastbar, nicht hörbar u. s. w. und Gesehenes, Getastetes und Gehörtes u. s. w. sind die Objekte der Naturwissenschaft. Neben diesen Farben, Tönen u. s. w. aber, die in räumlich-zeitlicher Ordnung, zu Komplexen vereinigt, der Umschau begegnen, besteht noch ein Wechselspiel von Gedanken, Gefühlen und Begehrungen, die in gleicher Weise der Betrachtung zugänglich sind und mit jenen körperlichen Erscheinungen in bestimmten Beziehungen stehen. Auch die Gedanken, Gefühle und Begehrungen weisen keinerlei Merkmale auf, die dem Naturforscher ihre Deutung als bloßer "Erscheinungen mit substanzieller Unterlage" nahelegten, sie beschïftigen ihn vielmehr analog dem Gesehenen oder Gehörten. Körperliche wie geistige Erscheinungen stehen dem Naturforscher als Tatsachen gegenüber, mit beider Erklärung hat der Naturforscher zur Befriedigung seiner praktischen und intellektuellen Bedürfnisse zu rechnen und sein Ziel ist daher nur: beide in kontinuierlicher Erscheinungsreihe darzustellen und ihrer schein. baren Unregelmäßigkeiten zu entkleiden, nicht aber darüber hinaus Reflexionen über allenfalls zugrunde liegende "Dinge“ und "Seelen" anzustellen.

Die hier gezeichneten "Grenzen" zu beachten, 
wird der Naturwissenschaft also keinesfalls mehr besonders beschwerlich fallen. Dagegen $\mathrm{muf}$ sie mit der größten Sorgfalt den Standpunkt bestimmen, den sie gegenüber dem Faktum einer Körper-und Gedankenwelt einnehmen soll, nachdem sie über die materialistische gănzliche bezw. teilweise Ignorierung der letzteren hinausge. kommen ist. Welches ist die Relation zwischen den beiden Welten? Affizieren sie sich gegenseitig? In gleicher Weise, oder in einseitiger Überordnung des einen Faktoren? Affizieren sie sich nicht und sind ihre Beziehungen nur solche der Beiordnung zweier gleichwertiger und gleichberechtigter Reihen des Geschehens in der Wirklichkeit?

In der Besinnung auf die Eigenart ihrer Erkenntnisse gewinnt sich die Naturwissenschaft hier ihren Standpunkt.

\section{III}

\section{Die naturwissensehaftliche Erkliirung der willkiirlichen Bewegung.}

Hierbei beeinflußt sie vor allem der Gedanke an die Befriedigung des Kausalitätsbedürfnisses: der Stützung schwächerer Gedanken durch stärkere (M a ch).

Die Einverleibung der vorliegenden Vorgänge in umfassendere und schließlich in allgemeine 
Reihen des Naturverlaufs ist beendet mit der Aufzeigung der einen universellen Gesetzmäßiglieit. In der physikalisch-chemischen, wie physiologischen Welt besitzen wir nun eine allem Anscheine nach lückenlos in sich zusammenhängende Kausalitätsreihe, der nach der mechanistischen, wie energetischen Körperweltstheorie nur relative Verschiebungen von Massen, bezw. Energicen zugrunde liegen; ein regelmäßiger Zusammenhang der physischen mit den psychischen Vorgängen ist offenbar vorhanden. Indiziert nun dieser Zusammenhang eine für beide Welten gemeinsame Kausalreihe, deren Glieder abwechselnd physisch wie psychisch bedingte darstellen können, oder deutet der Zusammenhang auf eine neue unabhängige Kausalreihe, die gegen die physische in sich abgeschlossen ist, und statt durch ein kausales, durch ein parallelistischesVerhältnis an jen e geknüpft ist?

Im ersteren Falle sieht sich die Naturwissenschaft vor die Tatsache gestellt, daß Druck und Stoß, bezw. Energieverschiebungen, nicht genügen, eine lückenlose Kausalerklärung der körperlichen Vorgänge zu liefern, sondern daß sie zur Ergänzung psychischer Erklärungsfaktoren bedürfen. Ein physischer Vorgang kann dann ebenso die Wirkung eines psychischen sein, wie seinerseits psychische Vorgänge im Gefolge haben. Im anderen Falle sind beide Welten 
inkommensurable Größen, die als Folgerung aus ihrem korrespondierenden Verlauf eine parallelistische Theorie als Deutung nahelegen. Nochmals sei hier der empirisch bedingte Charakter der naturwissenschaftlichen Erkenntnisweise betont, der nur in negativer Hinsicht von metaphysischer Tragweite sein kann - insofern er gewisse metaphysische Spekulationen ausschliebt -, ohne aber selbst etwa zugrunde liegendes transzendentes Terrain zu beanspruchen.

Die Hauptmomente, die für die Kausalitätstheorie, die Lehre von der Wechselwirkung zwischen Leib und Seele, in Betracht kommen, wollen wir in folgendem einer Untersuchung unterziehen nach dem Wert oder Unwert, den sie für eine Berücksichtigung als Erklärungsfaktoren innerhalb einer im übrigen mechanischen Reihe der Körperwelt beanspruchen können.

Für die Zwecke der folgenden Ausführungen ist die - je nach der Art der diskutierten Fragen - einmal stärkere Betonung der energetischen, dann der mechanistischen Weltanschauung als der körperwissenschaftlichen ohne Belang. Wie wir noch unten sehen werden, kommen für uns allein die übereinstimmenden Gedanken beider Theorieen in Betracht (Veränderungen in den äußeren Relationen); ihre Differenzpunkte sind zunächst von untergeordneter Bedeutung. 
Im Anschluf an Ausführungen Sigwarts ${ }^{1}$ ) und Stumpfs${ }^{2}$ ), welche die Geltung des Gesetzes der Erhaltung der Energie für rein quantitative Verhältnisse betonen, für welche psychische Geschehnisse ebenso gut wie physische Umsetzungen das Quantum abgeben künnten, findet sich bei Busse (die Wechselwirkung zwischen Leib und Seele etc. S. 99) folgende Formulierung des Versuchs einer Verbesserung bezw. Erweiterung des mechanistisch-energetischen Weltbildes: „Nicht der physische Kosmos allein, sondern das Geistiges und Phy sisches in sich enthaltende und zu mannigfacher Wechsel wirkung miteinander verknüpfende Universum bildet . . . eine in sich geschlossene Totalitä; nicht die Summe der physischen, in ihm enthaltenen Energie ist konstant und keiner Verminderung und Vermehrung fähig. Aller Austausch von Energieformen, sowohl der physischen und psychischen untereinander, als auch der physischen mit psychischen

1) „Das Gesetz der Erhaltung der Energie betrifft nur die quantitativen Verhältnisse und besagt, daß, wo ein Wirken stattfindet, diese quantitative Gleichheit besteht ... zwischen der Wirkungsfïhigkcit, welche ein Körper gewinnt, und der, welche der andere verliert.

2) "Läßt das Gesetz der Erhaltung der Energie, so argumentiert Stu mpf, die Natur der sich nach ihm ineinander umsetzenden Energieformen gänzlich unbestimmt, so hindert uns nichts, ebenso wie Wärme, Elektrizität etc., auch das Geistige als eine besondere Energieform anzusehen u. s. w." (Busse, a. a. O. S. 98 f.). 
vollzieht sich nach dem Prinzip der Äquivalenz, für jedes irgendwo verschwindende Quantum physischer oder psychischer Energie erscheint anderswo ein anderes genau entsprechendes Quantum physischer oder psychischer Energie."

Diese Formulierung des Energiegesetzes, wonach die physische Energie nicht mit Ausschluf, sondern in Gemeinschaft der psychischen die Kausalreihe darstellt, ist die ihren konträren Standpunkt am konsequentesten durchführende Theorie, dennoch aber, wie wir sehen werden, immer noch haltbarer als scheinbar weniger abweichende Varianten, die aber dafür den Sinn des energetischen Prinzips gewaltsam verzerren. Nach ihr hätten wir uns etwa folgende Vorstellung vom Ablauf einer willkürlichen Bewegungshandlung zu machen: Das Bild des ertrinkenden Kindes gelangt auf zentripetalen Leitungsbahnen, auf chemisch-physilkalisch erklärbaren Wegen, zum Gehirn; psychische Umsetzungen verarbeiten das nervös zugeleitete Material und effektuieren "die Strebung": das Kind zu retten. Die Strebung lädt ihre Energie auf die motorischen Bahnen über und auf chemisch-physikalischem Weg erfolgt wieder die Ausladung der nerrösen physischen Erergic durch die Arbeit der innervierten Muskelbezirke. Wir unterscheiden also eine zweimalige Formenänderung der quantitativ gleichbleibenden Energie. Eine physische Energieform besteht er'st bis zum vollendeten Ablaufder'sen- 
siblen Erregung, bei ihrem Abbrechen beginnt das Spiel der Empfindungen, Vorstellungen u. s. w. Form der psychischen Energie, - diese überläBt wiederum in der willkürlichen Bewegung das Feld der Form der körperlichen Energie.

Eine derartige Ausdehnung des energetischen Prinzips auf die Welt des Seelischen läßt sich mit der von seiten der körperlichen Naturwissenschaft vorgenommenen Interpretierung des Energiegesetzes, die sich a ufForschungsergebnisse stützt, nicht vereinbaren. Nicht nur, weil etwa die Anwendung der mechanistisch-energetischen Naturbetrachtung auf das gesamte Bereich der körperlichen Naturvorgänge bisher genügend erfolgreich gewesen wäre und die Berücksichtigung eines neuen Gesichtspunktes überflüssig erscheinen ließe, sondern vor allem aus der Erwägung heraus, daß für die naturwissenschaftliche Forschung sich bisher eben noch keine Nötigung ergeben hat, für physische Vorgänge nach nichtkörperlichen Erklärungsgründen zu fahnden, vielmehr noch jeder Naturvorgang, (solange nicht nach seinen metaphysischen etc. etc. Bedingungen gekundschaftet wurde, bezw. solange sich die objektivierende Betrachtungsweise von der historischen individualisierenden freihielt), bei $\mathrm{An}$ wendung der der Naturwissenschaft zu Gebote stehenden Hilfsmittel sich der 
Einreihung in den physischen Kausalzusammenhang gefügt hat.

Ziehen wir das oben erwähnte Beispiel der willkürlichen Bewegung heran, so mag ja allerdings beim ersten Anschein die unmittelbare Erfahrung für die Unterbrechnng der sonst gesetzmäßigen kontinuierlichen Reihe der körperlichen Vorgänge sprechen. Ein wenn auch noch so kurzer psychischer Akt scheint sich als bedingtes und bedingendes Glied in eine nicht mehr kontinuierliche Reihe physischer Glieder einzuschieben. Doch bedarf ihrer der Naturforscher zur Er. klärung der resultierenden willkürlichẹn Bewegung?

Er vermag die kontinuierliche Kette des Vorganges auch mit rein physisch bedingten Gliedern, ohne Zuhilfenahme jener darzustellen. Die gleichmäßige Einbeziehung der physischen und physiologischen Umsetzungen unter das Gesetz der Erhaltung der Energie darf hierbei keinen Anstoß erregen. Die bisher geleisteten Untersuchungen ${ }^{1}$ ) über die Energieaufnahmen und -ausgaben im organischen Körper, die u. a. in Rubners und Voits Laboratorium wertvolle Resultate gezeitigt haben, ergeben in dieser Richtung eine vollständige Parallelität der organischen und anorganischen Erscheinungen. B. Erdmann teilt uns in seinen "Wissenschaft-

1) S. a. o. S. 19 . 
lichen Hypothesen über Leib und Seele", S. 111, folgendes Ergebnis der diesbezüglichen Forschungen des amerikanischen Physiologen W. O. Atwater mit: "Führt man die Resultate dieser Experimente auf ihre einfachsten Formen zurück, so zeigen sie, daß die vom Körper in den zwei Formen von Wärme und äußerer Muskelarbeit abgegebene Energie der potentiellen Energie des oxydierten Stoffes gleichkommt. Die natürliche Schlußfolgerung besagt, daß̊ tatsächlich alle in (unserem) Körper umgewandelte Energie als Würme oder als Wärme mit äußerer Muskel. arbeit erscheint. Dies stimmt mit dem Gesetz der Erhaltung der Energie überein" ${ }^{1}$ ).

Um auf die Lücke zu stoßen, die den im übrigen zusammenhängenden Ablauf der physikalisch-chemischen Bewegungsvorgänge unterbricht und zu ihrer Erklärung notwendig einsetzender Bewußtseinsvorgänge bedarf, wollen wir die willkürlichen Bewegungen - etwa bei der überlegten Rettung eines ertrinkenden Kindes - in der Weise betrachten, daßs wir für

2) Der hier naheliegende Gedanke, wo denn dann die psychische Energie verbleibe, bezw. zum Vorschein komme, wird auch dort erwogen: Jede ihr entstammende Energiemenge mußte, "da sie in den Versuchen nicht zum Vorschein kam, entweder äußerst klein gewesen sein, oder es mußte sich um ein Etwas handeln, dessen Natur uns durch physikalische Forschung noch nicht enthüllt worden ist" (a. a. O, S. 112). 
jeden erfolgenden physischen Prozeß seine äquivalenten Ursachen aus dem Bereiche des Körperlichen uns darzustellen bemühen.

Die Erklärung der schon äußerlich sichtbaren Kürperbewegungen auf Grund mechanischer, bezw. physiologischer Einwirkungen dürfen wir hierbei, soweit exakt meßbare Muskel- und Nervenprozesse in Frage kommen, als gegeben erachten.

Spielt jedoch der Mechanismus unseres Nervensystems, wie er prompt und meßbar in den unwillkürlichen (Reflex-)Berregungen abläuft, auch hierbei dem willkürlichen Bewegungsakt, wo zwischen den physischen Vorgängen der optischen etc. Einwirkungen des Ereignisses und der Ausführung der Rettung die psychologischen Tatsachen als erste sich aufdrängen?

Mit der Wahrnehmung jagen sich die Vorstellungen der Pflichten gegen sich und Familie und der Pflicht dem Kind das Leben zu retten u. s. w., ein e Vorstellung behauptet das Terrain und motiviert den Willen, der Entschluß effektuiert die mit physischen Kräften erfolgende Rettungshandlung.

Aus dem Heer der psychischen Vorgänge, die in solchen kritischen Lagen in Frage kommen, nehmen wir einige heraus und legen ihnen die Hauptrolle für die Willensmotivierung bei. Bei der Wahrnehmung des ertrinkenden Kindes trete erst der Gedanke auf: rette das Kind; dann: 
erhalte dich selbst; schließlich der motivierende: du rettest womöglich das fremde und erhältst dich gleichzeitig. Die Gedanken sind stark gefühlsbetont und beherrschen in dieser Gefühlsbetonung (vor allem) den Ablauf der psychischen Vorgänge. Diesen psychischen Vorgängen, denen der Naturforscher keinen Einfluf auf die physischen einräumen kann, wollen wir nun - für die naturwissenschaftliche Erklïrung genügende

- Nerven- bezw. Gehirnprozesse zugrunde zu legen versuchen. Wir entwickeln die hier hereinspielenden physiologischen Vorgänge (die in unserem Falle mit den Gedanken an das Wohl des fremden und eigenen Kindes u. s. w. einhergehen) am Meynertschen Schema der Analyse des Vorganges, wie das gebrannte Kind dazu kommt, das Feuer zu scheuen. Psychologisch betrachtet haben wir beim erstmaligen Gebranntwerden des Kindes folgende Momente zu unterscheiden:

1. Eine Lichtempfindung (von dem Kerzenlicht ausgehend),

2. eine Bewegungsempfindung (beim Greifen nach dem Licht),

3. ein Unlustgefühl (beim Gebranntwerden),

4. eine Bewegungsempfindung (rasches Wegziehen der Hand),

5. ein Lustgefühl.

Der ganze Vorgang bietet der Aufstellung einer physiologischen kontinuierlichen Reihe 
keine Schwierigkeiten. Den fünf unterschiedenen psychologischen Hauptmomenten entspricht:

1. der sensible optische Vorgang (im nervus-, thalamus opticus etc.),

2. die reflektorisch erfolgende Greifberwegung (nach dem Licht),

3. die auf sensiblen Bahnen geleitete Nervenerregung (beim Gebranntwerden),

4. die wieder auf reflektorischem Wege erfolgende Abwehrhewegung,

5. der Befreiungszustand der Hand.

Nach wiederholten schlimmen Erfahrungen wird es das Kind vermeiden dem Licht zu nahe zu kommen, indem es in einer willkürlichen Bewegung die allenfalls schon genäherte Hand zurückzieht. Im psychologischen Tatbestand haben wir dann folgende Veränderung zu konstatieren: Auf die Lichtempfindung folgen nicht mehr der Reihe nach verschiedene Bewegungsempfindungen und Lust- und Unlustgefühle, sondern entsprechende gefühlsbetonte Vorstellungsvorgänge, als deren Effekt dic Abwehrbewegung resultiert. Die physiologischen Parallelvorgänge, an die sich der Naturforscher hält, sind jetzt außer dem Optikusreiz und der schließlichen, durch motorische Innervation bedingten, Abwehrbewegung Gehirnprozesse, die sich zwischen die eben genannten Geschehnisse sensibler und motorischer Natur einschieben. Die durch Optikusreizung hervorgerufene Erregung des Sensoriums muß 
auf kürzeren Bahnen, die beim erstmaligen diesbezüglichen Erlebnis noch nicht vorhanden waren, sondern erst geschaffen, bezw. ausgeschliffen werden mußten, sofort zur Bewerkstelligung der Endbewegung übergehen, während sie früher eines mehrfachen Reflexvorganges zu diesem Zwecke bedurfte. In der Tat sind wir auch, infolge der ergebnisreichen Untersuchungen Flechsigs und Verworns über die Assoziationsbahnen, nicht mehr genötigt, psychische Vorgünge als Lückenbüßer zur Erklärung einer auf den ersten Anschein hin neu aufgenommenen zweck. mäßigen Handlung heranzuziehen. Anatomischentwicklungsgeschichtliche und linische Erfahrungen haben uns wertvolle Aufschlüsse erteilt über jene kürzeren Bahnen, welche die physiologischen Äquivalente der psychischen Vorgänge darstellen, und welche über große, bisher noch unerforschte Großhirnindenbezirke verteilt sind. Bekannt ist, daßs sich die Nervenfasern der Großhirnrinde nach Anordnung und Richtung in zwei Klassen trennen lassen. Während die einen, vorwiegend den motorischen und sensorischen Bezirken angehörenden Fasern in ihrem Verlauf der Gehirnoberfliche zustreben, zeigen die anderen einen mit der Oberflïche mehr oder minder parallelen Verlauf ihrer Richtung. „Diese Fasern wurden von Flechsig als "Assoziationsfasern" bezeichnet, weil sie die einzelnen Oberflächengebiete der Hirnrinde miteinander verbinden. 
Flechsig hat nun die Beobachtung gemacht, daß in der Entwicklung des Gehirns sich eine Verschiedenheit zwischen beiden Faserarten bemerkbar macht. Die Nervenfasern der sensorischen und motorischen Sphären sind schon bei der Geburt im wesentlichen fertig entwickelt. Sie sind schon „reif", d. h. sie haben sich bereits mit einer Markscheide umhüllt. Die Fasern in den Assoziationsgebieten dagegen reifen erst nacheinander im Laufe vieler Monate. Darin liegt ein wesentlicher Unterschied. Und wenn wir dementsprechend die geistige Entwicklung des Kindes betrachten, so finden wir folgendes: Schon nach der Geburt sind die Muskeln und die Sinnesorgane tätig. Die motorischen und sensorischen Sphären können bereits funktionieren. Was aber beim Kinde noch fehlt, ist das höhere geistige Leben: die Vorstellungs-, Gedanken- und Urteilsbildung. Diese entwickelt sich erst im Laufe der Zeit. Und so liegt der Gedanke nahe, daß3 die Assnziationssphären Flechsigs in engstem Zusammenhang stehen mit den komplizierten Bewußtseinsvorgängen. Es hat sich dementsprechend auch durch eine Reihe von pathologischen Erfahrungen nachweisen lassen, daß Zerstörungen von gewisser Ausdehnung in diesen Gebieten mit entsprechenden Ausfallssymptomen im Geistesleben der betreffenden Personen verbunden sind. Wir beobachten in solchen Fällen bestimmte Intelligenz- 
defekte, und zwar von sehr charakteristischer Form" ${ }^{1}$ ).

Die eben gemachten Erlïuterungen schaffen gewiß nicht alle Schwierigkeiten bezüglich der physiologischen Deutung des gewählten Beispieles einer willkürlichen zweckmäßigen Handlung hinweg. Die übrigbleibenden Schwierigkeiten sind jedoch keine unüberwindlichen und nur empirischer Natur. Wenn die zugrundeliegende Frage "trotzdem bis jetzt sich nicht in bestimmter Weise hat beantworten lassen und auch wohl in absehbarer Zeit nicht beantwortet werden wird, so liegt das (nicht an ihrem transzendenten Charakter), sondern an der Unvollkommenheit der experimentellen Hilfsmittel, welche den ungeheuer verwickelten Lebenserscheinungen gegenüber versagen" ${ }^{2}$ ).

Es versteht sich nun auch ohne weiteres, daßf für den Naturforscher die Unterscheidung zwischen willkürlichen und unwillkürlichen Bewegungen keine wesentliche ist. Auf den ge-

1) M. Verworn, Die Mechanik des Geisteslebens. 2. Aufl. S. 61f. Im AnschluB daran stellt Verworn die Frage: "Wie kommt nun ein cinfacherer oder ein komplizierter Bewußtseinsakt durch das Zusammenwirken dieser verschiedenen Sphären zustande?" Für die heute nicht mehr materialistische Naturwissenschaft ist sic selbstverständlich gegentsandslos. Der Physiologe leitet aus dem Zusammenwirken dieser verschiedenen Sphären nur Nervenund Muskelprozesse u. s. w. ab.

$\left.{ }^{2}\right)$ E. König, Z. f. Ph. u. ph. Kr. Bd. 119, S. 25. 
gebenen nervösen Reiz folgt für ihn automatisch die einzig mögliche Reaktion und eine mehrfache Reihe solcher Realstionen, die vorerst allerdings zum großen Teil exakte Messungen und Nachweise noch nicht gestatten, (und die unbeschadet der Theorie auch für immer in ein teilweises exegetisches Dunkel gehüllt bleiben dürfen), ergibt für ihn die physische Handlung.

Wir haben uns nun den physiologischen Vorgang bei unserer willkürlichen Rettungshandlung vorzustellen ${ }^{1}$ ).

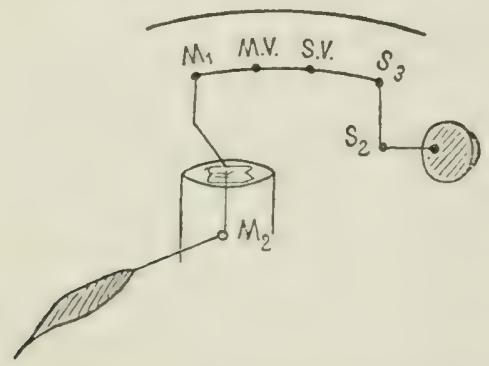

Die Veranlassung zurHandlung gab in unserem Beispiel die Wahrnehmung des ertrinkenden Kindes. Welches sind nun die nächsten Vorgänge? "Wo wir im Anschluß an eine Sinnesempfindung eine willkürliche Bewegung ausführen, da wird sich auf der Bahn der Sinnesleitung zunächst derselbe Erregungsvorgang abspielen ...,

1) Wir benützen zur Verdeutlichung eine (abgeänderte) mit beigegebener Erläuterung versehene Zeichnung Verworns (Mechanik ... S. 74 f.). 
aher er wird nicht in den Zellen der Sehsphäire endigen (Fig. S. 3), sondern er geht von den Zellen der Sehsphäre durch die tangentialen Assoziationsfaserbahnen nach einem Zentrum hin, das zu den Assoziationsgebieten gehürt (Fig. S.V.). Durch die Erregung der Zellen dieses Zentrums entsteht ${ }^{1}$ ) die Vorstellung oder die Kette von Vorstellungen, die Überlegung u. s. w., die der Ausführung der Bewegung vorangeht. Von den Ganglienzellen der an dieser Überlegung heteiligten Assoziationsgebiete wird damn der Impuls weiter befördert nach dem Teil des Assoziationsgebietes, in dem die Vorstellung der auf die gegebene Situation passenden motorischen Reaktion gebildet wird (M. V.) und ron diesem Bewegungsvorstellungsgebiete gelangt die Erregung erst nach der motorischen Sphäre selbst. Dort wird dann wieder eine bestimmte Zellengruppe in dissimilatorische Erregung versetzt und pflanzt den Impuls auf dem Wege ihrer langen Nervenfasern durch die ganzen tieferen Teile des Gehirns hindurch bis zu einer bestimmten Partie auf der gegenüberliegenden Seite des Rückenmarks fort $\left(\mathrm{N}_{2}\right)$, wo wiederum Zellen erregt werden, die den Impuls durch ihre Nerven schließlich zu den Muskeln hinleiten ..."

$\left.{ }^{1}\right)$ Der Nichtmaterialist kann sich selbstverständlich mit diesem ,entsteht" nicht einverstanden erklären. Die diesbezügl. Auseinandersetzung s. o. 
Die kompliziertesten willkürlichen Handlungen sind so nach ihrer physiologischen Seite hin naturwissenschaftlich $\mathrm{zu}$ analysieren. Und so rasch, bezw. bequem ihre Aufklärung durch psychologische Momente auch oftmals erschiene, die körperliche Naturwissenschaft braucht ihret. halben ron ihrem obersten Prinzip der durchgängig mechanistischen Erklärung nicht abzugehen. Sie hat „die Berechtigung, die verschiedenen Stufen geistiger Tätigkeit ron der einfachsten Reflexerscheinung his zur hüchsten Denkleistung als cine zusammenhängende Reihe zunehmend mannigfaltigerer und zweckmäßigerer Tätigkeiten anzusehen, die von der gleichen physiko-chemischen und physiologischen Grundlage ausgehen" (Ost. wald, Grundriß der Naturphilosophie S. 184).

Das Geständnis, das wir insofern machen müssen, als die unmittelbare Erfahrung bezüglich der Aufklärung des psychophysischen Zusammenhanges unserer Theorie nicht durchweg günstig gegenübersteht, kann die Naturwissenschaft in der Behauptung ihres Standpunktes nicht wankend machen. Denn die Vertreter der Wechselwirkung können sich nie in der Festhaltung ihres metaphysisch begründeten Standpunktes beruhigen und fühlen sich immer wieder veranlabit, den Anschluß an die gesicherten Ergebnisse der Naturwissenschaft zu suchen. Ihre diesbezüglichen Bemühungen scheitern jedoch alle an den nichtempirischen Voraussetzungen, die sie allzugewagte 
Hypothesen formulieren lassen. Die Ansicht Külpes, wonach bei der Annahme einer Äquivalenz zwischen den geistigen und materiellen Prozessen das Energiegesetz nicht beeinträchtigt würde, wenn geistige Energie sich in materielle Prozesse einschöbe, die Stumpfsche Theorie, wonach dasseibe Gesetz nicht verletzt würde, wenn psychische Vorgänge neben den physischen aus Nervenprozessen hervorgingen, ähnliche Ansichten Rehmkes und Wentschers sind sämtlich in gleicher Weise verzerrte Modifikationen des Mayer-Helmholtzschen Gesetzes der Erhaltung der Energie ${ }^{1}$ ). Nicht zuletzt auch Busses Interpretierung dieses Gesetzes, wonach zwar unbedingt zugegeben werden müsse, daß neue physische Energie durch physische Energie nicht entstehen könne, daß dies aber recht gut durch die Einwirkung psychischerWirkungen geschehen könne ${ }^{2}$ ). An der Auffassung, die Helmholtz, Mach etc. vom Energiegesetz besitzen, und die sie erst auf Grund empirischer, nachweisbarer Tatsachen gewonnen haben, kann durch "hypothetische Zutaten" zugunsten einer aus nichtempirischen Gründen verteidigten Wechselwirkung nicht gerüttelt werden.

$\left.{ }^{1}\right)$ Vgl. Busse, Die Wechselwirkung zwischen ... philosophische Abhandlungen (Sigwart-I3d.), 1900, S. $101 \mathrm{ff}$.

$\left.{ }^{2}\right)$ Ebenda S. 118. 
IV.

\section{Die mechanistisehe Yaturbetrachtmng mol das energetische Weltbild Ostwalds.}

Zu dem oben angeführten Resultat gelangt auch Os twald, jedoch nur unter einem ganz bestimmten Gesichtspunkt. Ostwald verwirft es „durch vorgefaßte Hypothesen, wie die mechanistische, die Forschung einseitig und für die abweichenden Tatsachen nahezu blind zu machen" und empfiehlt einfachere Wege, um „, für die Theorie der geistigen Erscheinungen einen stetigen und regelmäßigen Anschluf an die Theorieen aller anderen, insbesondere der Lebenserscheinungen zu finden ..." 1). Es ist vor allem der Verzicht auf "die Voraussetzung von der Gegensätzlichkeit der geistigen und der mechanischen Welt", an deren Stelle der Versuch zu treten hat, , eine Weltansicht ohne die Benützung des Begriffs der Materie ausschließ. lich aus energetischem Material aufzubauen" ${ }^{2}$ ). Hiermit versucht Ostwald die Aufstellung eines energetischen Weltbildes, wonach die beiden Energien des Körperlichen und des Geistigen gemeinsame Kausalreihen aufzustellen imstande wären. Der Unterordnung der beiden Begriffe Materie und

1) Grundriß d. Naturphilosophie S. 154.

$\left.{ }^{2}\right)$ Vorlesungen über Naturphilosophie S. 165. 
Geist unter den einen der Energie stand bis jetzt die Auffassung aller Energiearten als ausschließlich mechanischer gegenüber (Joule, Helmholtz), womit sich eine psychische Energie - soweit von dieser die Rede sein konnte - nicht gut vereinigen liebs. Die Folgen jener Auffassung bestehen nach Ostwald nun in unlösbaren Problemen, ,denen ein aufweisharer sachlicher Inhalt nicht entspricht", und die "am auffallendsten bei der wissenschaftlichen Behandlung der geistigen Erscheinung zutage treten". (Grundriß d. N. S. 152.) Prästabilierte Harmonie und psychophysischer Parallelismus sind danach fruchtlose Lösungsversuche jener Fragen, die aus der Überspannung des Gegensatzes der physischen und psychischen als mechanistischer und nichtmechanistischer Vorgänge entspringen.

In welcher Weise charakterisiert nun Ost. wal d die verschiedenen physischen Erscheinungen, um "den stetigen und regelmäßigen Anschluß für die Theorie der geistigen Erscheinungen" zu retten?

In der traditionell als Mechanik bezeichneten Wissenschaft ist als erste Unterseheidung die Trennung der Statik als der Lehre von der Arbeit und der Dynamik als der Lehre von der Bewegungsenergie und den Umwandlungserscheinungen der Arbeit in diese und umgekehrt vorzunehmen. Außerdem unterscheiden wir die Form-, die Volum- und Oberflächenenergic als mecha- 
nische, mit denen sich, nachdem ihre Bearbeitung früher als „Spielgebiet mathematischer Aufgaben betrachtet wurde", in neuerer Zeit in ausgedehnter Experimentalforschung die Elastizitätslehre, die Hydro- und Aeromechanik, die Kapillaritätslehre $u$. a. beschäftigen.

Damit scheint Ostwald das Gebiet der mechanischen Energieen abgrenzen zu wollen. Spezifische W eisen der Energie sind danachWärme, Elektrizität, Magnetismus, Licht und Chemismus, ferner die Erscheinungen psychischer Natur, deren energetischer Fassung, "zumal Denkarbeit ebenso mit Energieverbrauch und Ermüdung verbunden ist wie physische Arbeit" (Grundrils S. 188) keine Schwierigkeiten im Wegen stehen.

Von letzterem, der Möglichkeit der energetischen Fassung psychischer Er. scheinungen, vorerst abgesehen, scheint es nun doch gewagt, zugunsten einer ein. heitlichen Energetik mit kommensurablen Energiearten die Verschiedenheiten der mechanischen und nichtmechanischen übermäßig zu betonen, und dem Gegensatz physischer und nichtphysischer Formen gleichzustellen.

Nach der mechanischen Weltauffassung haben wir die Bewegungsvorgänge auf letzte Attraktionsuud Repulsivkräfte, deren Intensität durch rein räumliche Relationen von Atomen, Ätherteilchen, Elektronen etc. bedingt ist, zurückzuführen. Machen 
wir uns Ostwalds Auffassung zu eigen, so künnen wir auch noch der Ätherteilchen etc. wie irgendwelcher anderer hypothetischer Substrate entbehren, und uns nach der allgemeinen Energielehre mit reinen, trägerlosen Energieumwandlungen als eigentlich Seiendem begnügen. Die kinetische Energie ist demnach in mehr oder weniger verdichtetem Zustand über den Raum verteilt, der Begriff der Materie ein Relations. begriff aus Masse und Gewicht, die Masse der Zahlenfaktor zur Bestimmung der Kraft, die für bestimmte Körpergeschwindigkeiten notwendig ist $\mathrm{u} . \mathrm{s}$. w.

Wie unterscheidet sich nun die Bewegungsenergie von den übrigen physischen Energieen, etwa der Form der Wärmeenergie?

Die Gewißheit der Verschiedenheit besteht, "denn sonst könnte man sie eben nicht unterscheiden", wie auch "nur selten Zweifel bestehen, welcher Energieart man eine besondere Erschejnung zuordnen soll". (Grundriß S. 157.) Im übrigen aber - und nun kommt ein überraschendes Geständnis, nachdem man wohl die empirische Fundamentierung der Ostwaldschen Energetik vermutet hat, - konnte bisher "eine systematische Untersuchung der Mannigfaltigkeitscharaktere, durch welche sich z. B. eine Arbeit von einer Wärme u. s. w. unterscheidet und welches die wesentlichen, anderweitig nicht vorhan- 
denen Kennzeichen einer jeden einzelnen Energieart sind, noch nicht ausgeführt werden". (S. 157).

Als Sonderbeschaffenheiten bezeichnet denn Ostwald auch an der Wärmeenergie nur die auffällige physiologische Wirkung, sowie die Zugehörigkeit zu den einfachsten Energiearten, an der elektrischen und magnetischen Energie die polare Organisation ihrer „Eigenschaften“, den Mangel an Beziehungen zu physiologischen Sinnesapparaten (Vorlesungen über Naturphilosophie 2. Aufl., S. 228), an der chemischen Energie die große Mannigfaltigkeit der Eigenschaften und die geringe Gesetzmäßigkeit ihrer Beziehungen (Aufbewahrungsfähigkeit, Konzentration u. s. w.). Widerstehen nun diese Sonderheiten der einzelnen physischen, nichtmechanischen Energieen der Angliederung an die mechanischen in dem Maße, als das Wesen der psychischen Vorgänge mit dem der physischen kontrastiert?

Ostwald selbst bezeichnet als Charakteristikum der mechanisch interpretierbaren Energieformen die Möglichkeit der Anwendung räumlicher Begriffe auf ihre Veränderungen („verstehen wir darunter [unter mechanischen Formen] alle solche, bei denen mit räumlichen Änderungen Energieänderungen verbunden sind, so werden wir soviel verschiedene Formen zu erwarten 
haben, als räumliche Begriffe anwendbar erscheinen". Grundriß S. 154).

Und als Mannigfaltigkeitscharaktere der einzelnen physischen Energieweisen begegnen uns nun durchweg solche, die dieser Anwendungsmöglichkeit keine $\mathrm{Sch}$ wierigkeiten bereiten.

Weder die der Wärmeenergie als ein Spezifikum zugewiesene Wirkung auf den physiologischen Organismus, noch die Fals- und Leitbarkeit der elektrischen und magnetischen Energie u. s. w. widerstreben der Fassung ihrer Veränderungen im raum-zeitlich bedingte Gesetze, die chemische Energie in Sonderheit scheint auch weniger diesbezüglicher Bedenken wegen ilıre Sonderstellung gefunden zu haben, als wegen der psychologisch und methodisch verschiedenen Arbeitsweise von Physik und Chemie, ihres verschiedenen praktischen Wertes (s. u. a. Grundrifs S. 169). Kurz gesagt, die Sonderbeschaffenheiten, die Ostwald den einzelnen Energieformen zu ihrer Verselbständigung zuteilt, entbehren der Qualitaten eigenartiger, ihre Vereinigung zu ein- und derselben quantitativ bedingten Grundform nicht zulassender Erscheinungswesen.

Die atomistisch-mechanistische Kürperweltsauffassung bedarf zur Herstellung der den Bewegungen zugrunde liegenden Relationen, bezw. zur Vereinfachung der vorliegenden Mamnigfaltig- 
keiten durch die Relationsbegriffe, eines letzten rein quantitativen Dingbegriffes; dieses letzten Dingbegriffes glaubt die Energetik nicht zu bedürfen, da "Energie", nicht "Naterie", als das uns Gegenüberstehende, bezw. uns selbst Ausmachende zu gelten habe. Die Energie aber findet sich auch bei Ostwald wie an die Zeit so an den Raum gebunden, und mit dieser Gebundenheit muß sie sich wie die Materie den letzten begrifflichen Konsequenzen aus dieser Tatsache anbequemen. Die zeitliche wie die räumliche Natur der uns umgebenden Körperwelt fordern letzten Endes Unveränderlichkeit (und damit Unteilbarkeit) der letzten Dinge, rein quantitativ bestimmte, jeglicher Qualität entbehrende „Atom" begriffe. Selbst die letzte Vereinfachung zur Beseitigung der unübersehbaren Mannigfaltigkeiten kommt in der körperlichen Naturwissenschaft über die Anerkennung dieser Urteilchen nicht hinweg (vgl. Rickert, Grenzen der naturwissensch. Begriffsb. S. 85 ff.).

Auch Ostwald kommt darüber nicht hinweg, wenn er die Materie und die Körper energetisch zu interpretieren unternimmt. Wenn er etwa in der durch Betastung erfolgenden Konstatierung der Existenz eines "Körpers", die mit Recht als „eines der sichersten Kennzeichen für die tatsächliche Anwesenheit eines körperlichen Dinges angesehen wird" (O., Vorlesungen üb. N.-Phil. S. 169), vor allem nur die Anwesenheit von Volum- und $\begin{array}{llll}\text { Krug, Inaug.-Dissert. } & - & -49 & -\end{array}$ 
Formenenergie zu erkennen vermag, so ist eine derartige Feststellung vollständig unbrauchbar für eine Erleichterung des naturwissenschaftlichen Erkennens. Die "körperlichen Dinge", zu deren Anerkennung die Naturwissenschaft so oder so gezwungen wird, sind doch nicht mit einer Deutung an Be$r$ ührungs - und Druckempfindungen, an psychologischen Erklärungsfaktoren, hinwegdisputiert, in welcher Einkleidung hier Ostwald uns die Form-etc. Energien präsentiert.

"Die Behauptung Ostwalds, von materiellen Dingen wüßten wir nichts, wir erführen oder „fühlten" immer nur die Energie, führt gänzlich aus dem Gebiet der Physik hinaus, im günstigsten Falle zu erkenntnistheoretischen Deutungen, bei Ostwald aber zu sehr bedenklichen spiritualistisch gefärbten metaphysischen Behauptungen", schreilbt Rickert (in seinen Gr. d. n. Bgr. Bdg. S. 115). Sollte aber dem Wort "fühlen" die Bedeutung der Eliminierung des Körperbegriffs aus dem Inhalt des Gefühlten mit Unrecht untergeschoben werden, "nun, dann ist es lediglich als eine Änderung in der Terminologie zu betrachten, wenn man an Stelle des Wortes Materie das Wort Energie setzt... Die Energie ist dann nur ein neuer Name für das körperliche Ding, das als eigentliche Realitït der gesamten kürperlichen Energie zugrunde liegt" (S. 115). 
In der Fassung, wie uns Ostwald die Energetik darbietet, ist sie nicht in der Lage, die Naturwissenschaft in der mechanistischen Deutung aller physischen Vorgänge wankend $\mathrm{zu}$ machen. Ebensowenig ist aber auch die Berechtigung der Art und Weise anzuerkennen, in welcher Ostwald die Angliederung der psychischen an die physischen Vorgänge bewerkstelligt.

Da nach seiner Weltauffassung die Materie nur ein sekundärer Begriff ist, der dem primären Begriff der Energie "durch das konstante Zusammensein gewisser Energiearten" als Hilfsbegriff nachfolgt, besteht für den Energetiker von vornherein kein Gegensatz von Geist und Naterie, sondern nur das Problem des Modus der Beziehungen zwischen den psychischen Vorgängen und den verschiedenen physischen Energie. arten. Die parallelistischen Theorien erfahren eine kurze Ablehnung (denn es müßte z. $\mathrm{B}$. bei der unbegrenzten Umwandlungsfähigkeit der Energie und damit der Seele die Seelenbetätigung eines Milligramm Eisens eine ganz andere im menschlichen Blut als im Hufeisen sein (sic!), ferner müßte parallel der freien Energie „immer mehr Seele in den latenten Zustand übergehen, und die Weltentwicklung wäre daher in geistiger Beziehung als ein unaufhörlicher Rückgang aufzufassen ... Das widerspricht allen unseren Vor- 
stellungen von der Entwieklung..." $)^{1}$ ) mit Einwänden, deren schwache Stützen bezeichnenderweise Ostwald selbst gleich zugilbt (S. 375), allerdings mit dem Hinweis darauf, daß gegenüber dem Wirrsal und der Fragwürdigkeit der dem psycho-energetischen Parallelismus entspringenden Konsequenzen eine einfachere Lösung des Problems leicht zu finden sei.

Da nämlich einerseits „Veranlassung, die Wirkung eines seelenartigen Agens anzunehmen, ausschlieblich bei den Lebewesen vorliegt: ... die Theorie des psycho-energetischen Parallelismus diese Tatsache aber ungewürdigt lassen würde " 2 ), andererseits zahlreiche Analogien zwischen geistigen und körperlichen Erscheinungen der Erschöpfung $\mathrm{u}$. s. w. sich vorfinden, drängt sich die Vermutung auf: „daß es sich bei den geistigen Vorgüngen um die Entstehung und Umwandlung einer besonderen Energieart handelt" (S. 377).

Daß, wenn irgendein philosophischer Standpunkt dann sicher der hier bei Ostwald vorgefundene, der über die erkenntnistheoretische, bezw. logische Haltbarkeit der psychophysischen Theorieen nicht das geringste hesagt, mehr mit den Stützen des Gefühls als mit den Waffen des Nachweises errungen ist, ist nicht zu bezweifeln

$\left.{ }^{1}\right)$ O., Vorlesungen über N.-Phil. S. 374.

$\left.{ }^{2}\right)$ Vorlesungen S. 376. Die Behauptungen werden übrigens nicht begründet. 
und erwartungsvoll begrüßen wir daher die von Ostwald für nötig befundene Prüfung dieser Vermutungen auf ihre Vereinbarkeit einerseits mit den energetischen Grundgesetzen, andererseits mit den uns in den geistigen Vorgängen entgegentretenden Tatsachen.

Auf die Darbietung empirisch gestützter Tatsachen dürfen wir jedoch unsere Erwartung nicht einstellen.

"Wir können annehmen," versichert uns aufmunternd Ostwald (S.377), daß geistige Energie verbrauchter chemischer entspringt. Denn gemäß der Umwandlungstheorie geht ja mit geistiger 'T'ätigkeit und Erschöpfung proportional eine körperliche einher. Und nun folgt ein wiederholtes (inhaltlich materialistisch anmutendes) Spiel von "wenn" und "dann wäre". Also keine empirischen Tatsachen zwingen uns, sondern nur lose begründeten Annahmen finden wir uns nahegelegt!

Wir wollen gewiß nicht übersehen, dak auch die mechanische Körperweltstheorie in ihrer allgemeinen Ausdehnung nicht überall sich unumstößlicher Tatsachen als Grundlagen erfreuen darf, aber ihre Hilfshypothesen bewegen sich doch alle in dem einen Sinn der Reduktion der körper. lich en Mannigfaltigkeiten, wobei mit gleichem Maße meßbare Prozesse zur Verfügung stehen. Ostwald legt uns nahe, aus dem Analogon zweier Erscheinungen in völlig inkommensurablen 
Welten nicht bloß einer besonderen psychischen Energieart, sondern auch der Umwandlungsfähigkeit dieser in die physische Spezies zuzustimmen. Daß eine Hypothese von solcher Tragweite so sehr metaphysischer Stützen zu ihrer Aufstellung bedarf, läßt diesen Bestandteil der Ostwald. schen Energetik nicht recht überzeugend wirken. Vergebens suchen wir auch weiter nach Stützpunkten für Ostwalds Annahmen (auf die bekundete Absicht ihrer empirischen Prüfung hin), denn nach einer kurzen Vertröstung, daßi die Sache nicht für ganz hoffnungslos anzusehen sei ${ }^{1}$ ), „weil immer noch mit der Müglichkeit gerechnet werden darf, daß hinreichend entwickelte Meßmethoden zur Entscheidung der Frage gefunden und ausgebildet werden können", zieht Ostwald in weiteren gleich die Schlüsse aus seiner Annahme (S. 378).

1) Über die Art der den Konsequenzen entspringenden Schwierigkeiten vgl. „Es ist zurzeit noch keine Aussicht vorhanden, dies Experimentum crucis (Nachrreis, daß während des Denkens ein Anteil der gewöhnlichen Energicen verschwunden ist) mit Erfolg anzustellen, da insbesondere jede abgesonderte Handhabung etwa des Gehirns die vorherige Auslöschung aller geistigen Tätigkeit, d. h. den Übergang aller vorhandenen geistigen Energie in die gewöhnlichen Formen, zur Folge haben muß." S. 378. 
V.

Qualitative und quantitative, begriffliche und wirkliche Welt.

Zugunsten dieser Naturphilosophie ihre altbewährten und logisch durchaus zurechtfertigenden Forschungsprinzipien zu "verbessern“, wird sich die Naturwissenschaft der Körperwelt schwerlich herbeilassen. Was $\mathrm{K}$ ant der Psychologie in einer Mahnung an die Metaphysik zu denken gegeben („also muß empirische Psychologie aus der Metaphysik gänzlich verbannt werden"), das hat in gleicher Weise die Körperwissenschaft als W'arnung hingenommen und ist auch, wie es scheint, bisher der konkurrierenden Psychologie gegenüber glücklicher in ihren Bestrebungen gewesen. Sie ist auch, wie ihr billig zugestanden werden muß, nicht etwa auf der Flucht vor der Metaphysik, soweit von der Philosophie abgewichen, dak ihr System und ihre Forschungsmethode vor dem philosophischen Forum nicht bestehen könnten. Um so berechtigter darf sie sich bei ihrem empirisch und logisch wohlge. zimmerten Bau kritisch nicht geläuterte Systeme der Naturphilosophie vom Leibe halten.

Es darf nicht befremdend erscheinen, wenn es sich die Mehrzahl der Naturforscher so angelegen sein läßt, die nahe an Eliminierung grenzende Abschwächung des mechanischen Prinzips innerhalb 
des naturwissenschaftlichen Bereichs durch Ost. walds Energetik mit aller Kraft hintanzuhalten. Sie kann sich keineswegs mit Bus s es Anschauung befreunden, die mechanischen Bewegungsvorgänge seien letzten Endes nicht minder rätselhaft als etwa "die Annahme eines Austausches physischer und psychischer Energie und die damit verbundene Annahme einer qualitativen Energieumwandlung" 1 ).

Vor allem ist es der Naturwissenschaft um ein Verständnis, wie Busse es hier vor Augen hat, überhaupt nicht zu tun, und Busse insinuiert ihr ein Bestreben, das ihr seit den Zeiten des blühenden wissenschaftlichen Materialismus ferne liegt. Ihr "Erklären" will als das einer "Gesetzeswissenschaft" nicht weiter gehen, als bis sie das allgemeine Gesetz aus dem Chaos der Mannigfaltigheit herausgeschält hat. Über das in die Formen der Naturgesetze gefaßte Allgemeine hinaus auf transzendente Substrate zu fahnden, liegt der Naturwissenschaft fern, also auch - wie Busse dagegen zu glauben scheint über das metaphysische Wesen etwa der mechanischen Wechselwirkung Reflexionen anzustellen. Mechanische Bewegungen, wie sie den verschicdenen physischen Erscheinungsformen zugrunde gelegt werden künnen, fügen sich einer bestimmten einheitlichen begrifflichen Fassung, und sind da-

$\left.{ }^{2}\right)$ L. Busse, Die Wechselwirk, zw. L. u. S.... (S. 103). 
her dem Naturforscher der Körperwelt geeignetes Objekt. Psychophysische Kausalbeziehungen dagegen sind ihm nicht, „ebensowenig bezw. ebensogut verständlich", wie jene, sondern kommen, wie wir im folgenden sehen werden, für einen Vergleich, bezw. Angliederungsversuch (nach dem Muster der psychophysischen Wechselwirkung) für die körperliche Naturwissenschaft - gegenüber der Interpretation der Erfahrungstatsachen durch die Geisteswissenschaften überhaupt nicht in Betracht.

Wir wollen hier dem zunächst drohenden Einwurf begegnen, der Psychologie eigne doch als der Naturwissenschaft der geistigen Vorgänge dasselbe Prinzip wie ihren Schwesterdisziplinen: der gegebenen anschaulichen Mannigfaltigkeit gegenüber genau bestimmte allgemeine Begriffe zur Fixierung von Naturgesetzen zu formulieren. Woher der Kontrast in den Gegenständen ihrer Erkenntnisse?

Die in der logischen Begriffs- und Gesetzesbildung parallel gehende Erkenntnisart der Psychologie bedingt noch nicht eine sachliche Übereinstimmung der Eigentümlichkeiten beider Welten. Und diese sachliche Übereinstimmung beider wäre auch dann noch nicht vorhanden, wenn es der Psychologie gelänge, auch in dem einen Punkte der Zurückführung aller Mannigfaltigkeit auf einen einheitlichen einfachen Bestandteil den Vorsprung der körperlichen Naturwissenschaften 
einzuholen. Dieser letzte Bestandteil, der in der mechanischen Körperwelt einen unanschaulichen, rein quantitativen darstellt, bliebe vielmehr für die Psychologie immer ein unmittelbar auf das Bewußtsein bezogener, zeitlich-nicht räum. lich - bestimmter, anschaulicher Begriff. Die qualitative Beschaffenheit, die alles psychologische Geschehen um uns auszeichnet, verhindert jede Vergleichbarkeit des Seelischen und des Körperlichen.

Kommt diese schroff formulierte Gegensätzlichkeit nun für die Wirklichkeit in Betracht, nachdem sie nur a uf begrifflichen Konstruktionen beruht? Es besteht „unbedingt das Recht für die quantifizierbaren Elemente der Wirklichkeit den Namen den Physischen und für den nicht quantifizierbaren Rest den Namen des Psychischen zu verwenden, und diese beiden Gebiete zu besonderer Behandlung an zwei verschiedene Wissenschaften zu verteilen. Aber eine ganz andere Frage ist die, ob man die Produlste dieser Begriffsbildungen auch als für sich bestehende Realitäten ansehen und sie mit dem identifizieren darf, was wir als Geist und Körper unmittelbar erleben. Diese Frage ist schon aus dem Grunde zu verneinen, weil wir dadurch die Welt als Ganzes unbegreiflich machen" schreibt Rickert (in s. Abh. üb). psph. K. u. Par. Phil. Abh. 1900, S. 76). 
Gewiß, daß uns die Wirklichkeit in ganz anderer Weise als in letzten quantitativen oder qualitativen Bestandteilen gegeben ist, stelıt nicht in Frage. Es handelt sich aber für die Naturwissenschaft nicht nur um die Tatsachen, die uns beim unmittelbaren Erlebnis der Wirklichkeit gegenübertreten. Danach kann es ja keinem Zweifel unterliegen, daß qualitätslose Elemente in der unmittelbaren Erfahrung überhaupt nicht entdeckt werden können, sondern ihr Dasein nur begrifflicher Produktion rerdanken ${ }^{1}$ ). Dagegen ist sehr $\mathrm{zu}$ beachten, ob das menschliche Erkenntnisvermögen nicht automatisch zu jener Unterscheidung drängt, sobald statt der unmittelbaren Erfahrung ein Eindringen in ihr Verständnis beginnen soll.

In der unmittelbaren Erfahrung ist ein lebhaftes Mit- und Aufeinanderwirken physischer und psychischer Vorgänge gegeben, so daß eine durchgängige scharfe Trennung überhaupt sehr erschwert und mitunter unmöglich erscheint. Doch dieses einheitliche psychophysische Geschehen verändert sich sofort von Grund aus, wenn wir eine logisch begriffliche Verarbeitung beginnen wollen; dies ist aber jedesmal der Fall, wenn wissenschaftlichen Methoden in irgendeiner Materie zur Anwendung gelangen sollen. Die wissenschaftlichen Methoden der körperlichen Naturwissenschaft führen nun immer wieder

1) Siehe auch S. 62 f. 
zur Quantifizierung zurück und lassen die psychischen Geschehnisse wegen ihrer Sonderbeschaffenheiten als wesensverschiedene zurück. Wenn Rickert daher eingesteht, (Phil. Abh. s. o.): „daß der Begriff des Wirkens, den wir benutzt haben, um wenigstens die Möglichkeit psychophysischer Kausalität aufzuzeigen, in hohem Grade unbestimmt ist und uns nicht gestattet, mit seiner Hilfe in wissenschaftlicher Weise einen Vorgang als Ursache und einen anderen als Effekt aus der unübersehbaren Mannigfaltigkeit der empirischen Wirklichlieit eindeutig herauszuheben, wie dies durch die Aufstellung von Kausalgleichungen geschieht", so müssen wir hinzufügen, daß dieser der unmittelbaren Erfahrung entnommene Begriff in keiner Weise die aus der notwendig mechanistischen Deutung der kürperlichen Vorgänge hervorgegangene Überzeugung der Körperwissenschaft von der Unvergleichbarkeit der beiden Welten zu erschüttern imstande ist. Der Rickertsche Versuch, die in der unmittelbaren Erfahrung gegebene Welt in einer Weise zu deuten, „um das Feld frei zu machen für eine andere als mechanische Auffassung des Kausalverhältnisses", erscheint uns nicht aussichtsreich genug; denn mit dieser Betonung der unmittelbar gegebenen Erfahrung zum Zweck einer Wesensbestimmung der seelisch-körperlichen Beziehungen eröffuet sich das Feld für zu viele Spekulationen!

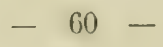


Primär erfaßite Erfahrungstatsachenzur Aufstellung von Theorieen über das Wesen physischer und psychischer Vorgänge und deren Zusammenhänge zu benützen, ohne gleichzeitig Erkenntnistheorie und Logik mitsprechen zu lassen, ist ein wenig Erfolg versprechendes Beginnen. Die in diesem Falle vorgefundene Unbestimmtheit spricht für alles und nichts und Spiritualismus und Kausalitätstheorie gedeihen darauf nicht besser und schlechter als Materialismus und Parallelismus. Sowie der Geist, bezw. die Wissenschaft sich auf „Erkenntnis" einzustellen beginnt, erfolgt aber eine trotz ältester Tradition noch immer von neuem sich aufdrängende erste Klassifikation der empirischen Beobachtungen, die wir mit der Unterscheidung der physischen und psychischen Welt statuieren.

Dieses Prinzip wird auch dadurch nicht in Frage gestellt, daß die einzelnen wissenschaftlichen Erkenntnisresultate mit den subjektiv bedingten Mannig. faltigkeiten, (mit psychologischen und historischen Bestandteilen) der Erfahrung vermengt sind.

Einesteils bedürfen ja große Gebiete der Naturwissenschaft restlos quantifizierbarer Resultate nicht (Botanik, Zoologie), und die Grundtendenz bleibt deshalb trotzdem noch unverkennbar. Andererseits verhehlt es sich z. B. die körperliche Naturwissenschaft der Biologie 
durchaus nicht, bei den gegenwärtigen Stand ihrer Forschungen eben von vorneherein auf bedeutende wünschenswerte Zergliederungen und Reduktionen noch verzichten zu müssen; sie verliert aber deshalb nicht die ihrer Disziplin gesetzten Aufgaben und Grenzen aus dem Auge, solange sie sich für diese noch nicht genügend erforschten Gebiete unter Vorbehalt subjektiver Charakterisierungen bedienen. Nach Rickert wäre es nun allerdings nicht möglich, „diese Begriffsbildungen nur als vorläufige anzusehen, die mit den Fortschritt der Wissenschaft verschwinden und durch rein quantitativ bestimmte Produkte ersetzt werden könnten. So lange es vielmehr Biologie und Chemie gibt, werden diese Wissenschaften es auch mit Qualitäten zu tun haben, und keine denkbare Vollendung der allgemeinsten Körpertheorie kann diese qualitativen Begriffe aufheben" (s. o. Philos. Abh. 1900).

Damit soll doch wohl nur die mensch. liche Unzulänglichkeit betont werden: die historisch überlieferten Verquickungen aller körperwissenschaftlichen Systeme mit psychologischen Begriffsbildungen und metaphysischen Gedankengängen jemals endgültig von den Lehrgebäuden der einzelnen Wissenschaften fernhalten zu können.

So lange wir uns inmitten der lebhaft fluktuierenden psychophysischen Wirklichkeit 
befinden, und physisch und psychisch ihrem Einfluß乃 preisgegeben sind, dürfte es freilich kaum gelingen, jene überkommenen Residuen reflexionsloser Feststellungen dauernd los zu werden. Für uns kommen aber bloß Er. wägungen logischer und erkenntnistheoretischer Art in Betracht, und da unterliegt es keinem Zweifel, daß eine möglicherweise ständige Verunreinigung chemischer etc. Wissenschaften mit Erklärungsfaktoren qualitativen Charakters kein Hindernis bildet, daß jene Wissenschaften im Sinne des logischen Prinzips sich entwickeln und schließlich das logische Ideal, bei allerdings formaler Unzulänglichkeit, verkörpern. Rickert selbst konstatiert (Grenzen ... S. 120) in der Chemie nicht blok das Streben nach Bildung eines Begriffs, „der die Aufstellung von unbedingt allgemeinen Sätzen über die chemische Hannigfaltigkeit ermöglicht, und es kommt in diesem Streben wieder genau die Tendenz zum Ausdruck, die wir aus logischen Gründen gefordert haben", sondern erkennt auch an, daß mit der Zurüclführung der Eigenschaften der Elemente auf die periodische Funktion ihrer Atomgewichte bereits ein Begriff gebildet ist, "unter den jedes Element sich als ein durch Atomgewicht, d. h. nur noch quantitativ bestimmtes Glied einer Reihe bringen läßrt". „Dann wäre auch die gesamte chemische Mannigfaltigkeit in einer rein mechanischen Naturauffassung 
untergebracht und dadurch vollstaindig begriffen". (S. 122.)

Hier kommt auch, indem jede Wissenschaft, wie wir oben sahen, noch mit Erklärungsfaktoren, die der empirisch gegebenen Mannigfaltigkeit entnommen sind, arbeiten muß, in Betracht, "dafs die verschiedenen Wissenschaften mehr oder weniger historische Bestandteile aufweisen". (S. 266.) Auch diese Tatsache kann nur besagen, daß die naturwissenschaftliche Betrachtungsweise in der Praxis nur schwer von der historischen sich trennen läßt - aus oben entwickelten Gründen -, sie ändert aber nichts an der sich bei jedem naturwissenschaftlichen Beginnen einstellenden Notwendigkeit: jene Unterscheidung an der Erfahrungswelt in psychische und physische Elemente vorzunehmen.

Eine andere Frage ist die, wie sich die Naturwissenschaft dem individuellen Wirken gegenüber, bei dem die körperlich-geistigen Beziehungen im Vordergrund stehen, grundsätzlich verhalten wird.

Selbstverständlich fällt es ihr heutzutage nicht mehr ein, das geschichtliche Individuum auf einen Mechanismus letzter quantifizierbarer Bestandteile zu reduzieren, wonach der Zusammenhang etwa von Klima, Rassentemperament und einer skandalösen Affäre in physikalisch-chemischen Formeln auf ein Blatt 
Papier projiziert werden könnte. So wenig ein verschachertes Linsengericht mit den politischen etc. Folgen von Jakobs Stammvaterschaft in Zusammenhang gebracht werden darf, so sehr wehrt sich heute die Naturwissenschaft gegen die Insinuation: in statistischen Aufstellungen das $\mathrm{A}$ und $\mathrm{O}$ individueller Geschehnisse und kultureller Motivierungen zu erblicken. Die Kulturwissenschaft hat sicherlich kein Bedürfnis nach naturwissenschaftlicher Nomothetik, noch darf sie überhaupt die Geschehnisse ihrer Qualitäten zu entkleiden suchen. Denn die Anwendung der Atomistik oder Energetik hier hieße mit Musik ein Gemälde schaffen wollen und wäre gleichbedeutend mit der Ignorierung aller erkenntnistheoretischen Elementarerrungenschaften. Man wird es der Natur. wissenschaft aber nicht verargen dürfen ${ }^{1}$ ), wenn sie in Erinnerung an die kläglichen Erfolge bei jeglicher Okkupation ihr nicht zugehörigen Terrains mit un verbindlichen Meinungsäußerungen sich bescheidet. Im Rahmen der naturwissenschaftlichen Erkenntnisweise läßt sich eine Erklärung für die Tatsache

1) Um so mehr, als ihr die Berechtigung sich in dieser Frage einzumengen von philosophischer Seite meist von vornherein aberkannt wird. 
der psychophysischen Welt nicht geben ${ }^{1}$ ).

Daran läßt sie aber nicht rütteln, daß eine psychophysische Theorie niemals berechtigt sein wird, Körperliches und Geistiges in einem Kausalzusammenhange im Sinne der Wechselwir. kungshypothese zu verbinden. Nach dieser Theorie ergeben sich Kausalungleichungen und mit solchen ist, wenn vielleicht auch innerhalb des historischen Wirkens, so doch nie im Be. reich der naturwissenschaftlichen Begriffs. und Gesetzesbildung etwas anzufangen.

Die philosophische Lösung aber, mit der allein sich die Naturwissenschaft zu. frieden stellen kann, "muß eine solche

1) „Von der Naturwissenschaft eine Lösung der in Rede stehenden Aufgabe verlangen heißt... von ihr fordern, daß sie neben den materiellen Elementen noch anderweitige Agentien einführe, daß sie also ihre Voraussetzungen über die letzten Grundlagen des äußeren Geschehens radikal umändere und die bisher verfolgten Bahnen verlasse. Nun bedarf es kaum der Erinnerung, daß die Periode des wissenschaftlichen Betriebes und des geregelten Fortschrittes der Naturstudien erst seit der Zeit datiert, wo der Grundsatz zur allgemeinen Anerkennung gelangte, daß alle ïußeren Vorgänge auch aus äußeren Ursachen, d. h. aus den konstanten Eigenschaften und Wirkungsweisen der materiellen Elemente zu erklären seien. Will man im Ernst diese ganze Entwicklung wieder rïckgängig machen und zu den Anschauungen der Naturphilosophen des 16. Jahrhunderts zurïckkehren? (E. König, Z. Ph. Kr. Bd. 115, S. 165.) 
sein, die das naturwissens chaftliche Weltbild unverändert läßt, und den Zusammenhang von Natur und Geist, das Nebeneinanderbestehen von mechanischer Kausalität und Zweckbestimmung vielmehr dadurch begreiflich zu machen sucht, daßs sie beide Formen des Seins und Geschehens auf einen einheitlichen metaphysischen Grund zurückführt. Wie ein Blick auf die wichtigsten philosophischen Systeme der Vergangenheit zeigt, ist dies in rerschiedener Weise müglich, sobald man nur einmal die Voraussetzung aufgibt, daß die äußere Wirklichkeit eine absolute Realität besitze, wogegen ron seiten der Naturwissenschaft nichts einzuwenden ist" (E. König, Z. Ph. Kr. Bd. 115, S. 165).

Nicht gering sind allerdings die Bedenken, die sich an die Annahme eines psychophysischen Parallelismus knüpfen. Ein universeller Parallelismus führt, wie Ricker't ${ }^{1}$ ) zeigt, zu Konsequenzen, die das Hauptmoment (Lnterscheidung qualitativer und quantitativer Welt) gefährden, um dessenwillen psychische und physische Vorgänge statuiert und getrennt wurden. Die Naturwissenschaft fühlt sich aber weder genötigt noch ermächtigt, über Resultate der empirischen Forschung hinaus den im Metaphysischen gelegenen Zusammenhang zwischen Körperlichen und Geistigen zu suchen; für sie kommt eine andere Parallelität als die auf unsere Individualität be-

$\left.{ }^{1}\right)$ Psychoph. Kausalität ... Philos. Abh. 1900, S. 70 f. 
zogene nicht in Betracht. Denn das Psychische ist ihr - wenigstens vorläufig - als ein individuelles gegeben und kann zum Körperlichen schlechthin nicht in Parallele gesetzt werden. Der Weg, der zur Ermöglichung der Relationshestimmung der beiden Welten eingeschlagen werden kann, darf über die Grenzen nicht hinausgehen, die ihm durch die notwendige Berücksichtigung des als individuell gegebenen Psychischen vorgezeichnet sind. Unter den physsischen Vorgüngen künnen daher zur Bildung der einen parallelen Reilhe nur jene in Betracht kommen, die ebenfalls individuell bestimmt sind. Münsterbergs Versuch, die Gehirnvorgänge als genügend für die Konstruktion des einen Faktors in den psychischen Bezichungen anzusehen, bekundet die Möglichkeit, dem individuell Psychischen eine physische Parallele entgegenzustellen. Soweit die physischen Vorgänge dann individuell sind, können ihnen die individuellen Vorstellungen zur Seite treten.

Von allerdings anderen Gesichtspunkten aus. gehend (zum Zwecke der Herstellung des psychischen Zusammenhangs) hat Münsterberg in diesem Sinne den psychophysischen Parallelismus als möglich, bezw. für die Logik der Naturwissenschaft als unumgänglich hefunden. $\mathrm{Ob}$ damit aber ein Schritt mehr zur Aufstellung eines allseitig befriedigenden philosophischen Weltbildes getan ist: Die philosophischen Ansichten stehen den Parallelismus in dieser wie 
in anderen Formen meist ablehnend gegenüber, da er der Berücksichtigung des unmittelbar Wirklichen zu wenig Rechnung trägt.

Die Naturwissenschaft kann nur betonen, daß mit einer Parallelismustheorie ihren eigenen Forderungen, die sie an die philosophische Lösung des Problems stellen muß, genügt werden kann, und daß ferner von parallelistischen Standpunkt aus sich manche Differenzen mit Tatsachen der Erfahrung vermeiden lassen. So beobachten wir ja allerdings bei der Willenshandlung das enge Verwachsensein des psychischen Aktes - der Konkurrenz der Gedanken um die Motivierung - und des physischen - der Handlung. Damit wird uns aber noch nicht die psychophysische Kausalitätstheorie anfgezwungen, wonach wir den psychischen Akt als die Ursache, den physischen als die Wirkung ansehen müßsten. Wir können die beobachtete physische Handlung als Effekt der nicht wahrzunehmenden Assoziations- und Innervationsrorgänge im Gehirn betrachten und stützen uns dabei auf Tatsachen der Erfahrung. Dabei verlieren wir nicht aus dem Auge, daß wir dem unmittelbar Wirklichen nicht gerecht werden, und der historischen bezw. psychologischen Betrachtungsweise nicht vorgreifen dürfen. Der Ausgleich zwischen Kausalzusammenhang und Zweckzusammenhang darf nur nicht mit der Einbuße der natur- 
wissenschaftlichen Kausalbetrachtung erkauft werden, und mag immerhin unter dieser Bedingung von einem metaphysischen Aktualitätsstandpunkt etc. aus in der Richtung einer voluntaristisch-teleologischen Wirklichlieitsauffassung erfolgen.

Im Widerstand gegen die Wechselwirkungshypothese bestärkt die Naturwissenschaft übrigens nicht eine besondere Vorliebe für den Parallelismus, dessen Ersatz durch andere der Naturwissenschaft gerecht werdende Theorien nicht grundsätzlich abgelehnt wird, sondern die Unvereinbarkeit der Annahme einer Wechselwirkung mit notwendigen wissenschaftlichen Objektivierungen. Was der Naturwissenschaft noch vor allem die Stellungnahme $\mathrm{zu}$ unserem Problem erschwert, ist die von seiten der Anhänger der Wechselwirkungshypothese vorgenommene Vermengung empirischer und metaphysischer Erwägungen. Darnach kann es ja nicht zweifelhaft sein, daß letztere gegenüber der mit empirischen Material arbeitenden Naturwissenschaft im Vorteil ist. Mit Recht bemerkt E. König $\left.{ }^{1}\right)$ : „Man will dadurch freie Bahn gewinnen, um unbehindert durch die Rücksichtnahme auf etwaige empirische Gegeninstanzen seine Hypothesen im Sinne rein spekulativer Erwägungen und Postulate gestalten zu können".

1) Warum ist ... zu verwerfen? Z. Ph. Kr. Bd. 119 S. 29. 
Die Hinmeise etc. beziehen sich auf folgende Literatur:

Rickert, Die Grenzen ... Begriffsbildung. - Psychophys.

Kausalität . . .; aus den Phil. Abh., Sigwart 1900

gewidmet.

Busse, Die Wechselw. zwischen L. u. S. . . Phil. Abh.,

Sigwart 1900 gewidmet. - Geist und Körper, Seele u.

Leib 1903.

Münsterberg, Psychologie.

B. Erdmann, Hypothesen über L. u. S. 1907.

Verworn, Mechanik des Geistesl., 2. A., 1910 (Natur und

Geistesw.).

F. Auerbach, Grundbegr. d. mod. Naturl., 3. A., 1910.

Bunge, Lehrb. d. path. . . Chemie, 2. A.

Lange, Gesch. d. Mat. II.

Drobisch, Neue Darstellung d. Logik, 5. A.

W. Ostiwald, Grundr. d. Naturph. (Reclam), Vorlesungen

ü. d. N., 2. A., Naturphilosophie in ,System. Phil.“ 1907.

E. König, Die Lehre vom psychophys. P. . . Z. Ph. Kr.

Bd. 115, Warum ist die Annahme einer psychophys.

Kausalität zu verwerfen? Z. Ph. Kr. Bd. 119.

Cornelius, Einleitung in die Philosophie 1903.

R. Hertwig, R. A. Rd. 1910/11.

A. Forel, Gehirn u. Seele 1910.

Bernheim, Lehrb. d. hist. Meth. 


\section{Lebenslauf.}

Ich, Wilhelm Krug, bin geboren am 23. Mai 1888 in Hohenwart, Kreis Oberbayern, als Sohn der Steuerverwalterseheleute Michacl und Katharina (geb. Kronwitter) Krug, bin katholisch getauft, bayerischer Staatsangehöriger. Vom 10. Lebensjahre an besuchte ich die Gymnasien in Ingolstadt, Dillingen und München (Ludwigsgymnasium). Mit 19 Jahren bezog ich die Hochschule und studierte Medizin und Philosophie an den Universitäten München, Rostock, Lausanne und Erlangen. 



\section{PLEASE DO NOT REMOVE CARDS OR SLIPS FROM THIS POCKET}

\section{UNIVERSITY OF TORONTO LIBRARY}

Krug, Wilhelm

Uber die Stellung der Naturwissenschaft zum Leib-SeeleProblem 
